\title{
Le tribalisme, stade ultime de la polarisation
}

Liliana Mason, Uncivil Agreement: How Politics Became Our Identity, Chicago, The University of Chicago Press, 2018 ; J.D. Vance, Hillbilly Elegy: A Memoir of a Family and Culture in Crisis, New York, HarperCollins, 2016

\section{Maya Kandel}

\section{OpenEdition}

\section{Journals}

\section{Édition électronique}

URL : http://journals.openedition.org/ideas/5526

DOI : $10.4000 /$ ideas.5526

ISSN : 1950-5701

\section{Éditeur}

Institut des Amériques

\section{Référence électronique}

Maya Kandel, «Le tribalisme, stade ultime de la polarisation », IdeAs [En ligne], 13 | 2019, mis en ligne le 01 mars 2019, consulté le 24 septembre 2020. URL : http://journals.openedition.org/ideas/5526 ; DOI : https://doi.org/10.4000/ideas.5526

Ce document a été généré automatiquement le 24 septembre 2020.

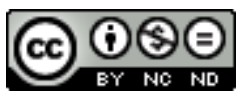

IdeAs - Idées d'Amériques est mis à disposition selon les termes de la licence Creative Commons Attribution - Pas d'Utilisation Commerciale - Pas de Modification 4.0 International. 


\section{Le tribalisme, stade ultime de la polarisation}

Liliana Mason, Uncivil Agreement: How Politics Became Our Identity, Chicago, The University of Chicago Press, 2018 ; J.D. Vance, Hillbilly Elegy: A Memoir of a Family and Culture in Crisis, New York, HarperCollins, 2016

\section{Maya Kandel}

\section{RÉFÉRENCE}

Liliana Mason, Uncivil Agreement: How Politics Became Our Identity, Chicago, The University of Chicago Press, 2018

J.D. Vance, Hillbilly Elegy: A Memoir of a Family and Culture in Crisis, New York, HarperCollins, 2016

1 Qu'est-ce que le tribalisme ? Faut-il voir dans ce mot utilisé pour décrire la nouvelle réalité politique américaine un effet de mode, typique des emballements de la bulle washingtonienne? Ou bien s'agirait-il d'un nouveau concept utile pour désigner le stade ultime de la polarisation du pays, qui serait donc quelque chose de différent?

2 Cette notion de "tribalisme" est, quoi qu'il en soit, de plus en plus utilisée à Washington, dans la presse ${ }^{1}$, dans les conférences ${ }^{2}$, dans les sondages, rapports et livres $^{3}$ - sans que l'on ne discerne dans son emploi, à première vue, autre chose qu'un nouveau degré dans la polarisation du pays. Pourtant, l'élection de Trump et le climat actuel aux États-Unis sembleraient indiquer qu'il se passe bien quelque chose de nouveau, que l'augmentation de la polarisation politique depuis près de trente ans a franchi un saut qualitatif pour produire un phénomène distinct. Il semble donc utile de se pencher sur ce fameux tribalisme, déjà repris sans plus de précision en Europe.

3 Les deux livres qui éclairent le mieux les États-Unis d'aujourd'hui n'emploient pas le mot tribalisme - mais illustrent le phénomène de manière éclatante : Uncivil Agreement, livre de la politologue Liliana Mason, professeur à l'Université du Maryland, publié 
cette année à partir de travaux de l'auteur depuis près d'une décennie; et la biographie familiale de J.D. Vance, Hillbilly Elegy, parue en 2016, best-seller souvent cité, avec raison, comme le livre à lire pour comprendre l'Amérique de Trump. La lecture croisée de ces deux ouvrages très différents donne peut-être le meilleur éclairage sur ce fameux « tribalisme » américain.

\section{Liliana Mason : le réalignement partisan des identités dans la société américaine}

4 Liliana Mason est une jeune politologue, maîtresse de conférences (assistant professor) à l'Université du Maryland, qui publie ici un ouvrage issu de ses travaux de thèse (donc bien avant que la notion de «tribalisme » ne devienne à la mode). Invoquant aussi bien la science politique et la sociologie que les sciences cognitives, de la neurologie à la psychologie sociale, l'ouvrage utilise force calculs et régressions mathématiques sur des chiffres venant d'instituts de sondages sérieux. Si la forme est parfois indigeste (comme beaucoup d'ouvrages américains contemporains de science politique, fortement mathématisés), l'analyse rigoureuse des chiffres est pour une fois largement justifiée, et permet à l'auteur d'asseoir solidement sa thèse. Le livre démontre ainsi comment les identités partisanes, idéologiques, religieuses et raciales des Américains se sont progressivement alignées (l'auteur utilise le terme "triées ", sorted), rendant chaque parti de plus en plus homogène en termes d'identité des individus.

5 La thèse du livre est que ce "tri », ou alignement, des identités par parti rend plus difficile toute forme de compromis politique entre les partis, et produit un électorat qui est à la fois plus biaisé, moins tolérant, et surtout facilement en colère vis-à-vis de tous ceux qui sont du bord politique opposé. En effet, cet alignement partisan des identités conduit à une simplification de l'identité sociale, qui a des effets psychologiques et politiques.

6 Pour les passionnés des questions politiques internes américaines, rappelons ici le débat récurrent, en arrière-fond de la polarisation politique croissante indéniable aux États-Unis, qui oppose depuis une quinzaine d'années les politologues Alan Abramowitz et Morris Fiorina (pour simplifier - chacun ayant ses partisans) (Mason L., 2018: 76-77) : plus précisément, Fiorina, fréquemment cité aux États-Unis et en France (il a par exemple été souvent invité pendant les élections américaines, notamment au CERI), explique, statistiques à l'appui, que ce ne sont pas les Américains qui sont polarisés, seulement la classe politique américaine. Intuitivement, surtout depuis Trump, on avait l'impression qu'il avait tort. Le livre de Mason démontre pourquoi.

7 Son livre démontre en effet que l'erreur des deux politologues est de ne prendre en considération que les opinions des Américains sur des positions politiques (policy). Or si l'affiliation partisane est devenue affaire d'identité, alors les positions politiques concrètes importent peu et peuvent même être modifiées au gré du positionnement du parti ou de son leader. La vie politique contemporaine américaine en fournit de nombreuses illustrations, du revirement des républicains sur la Russie ou le commerce, en phase avec les positions de Trump, au soutien des électeurs évangéliques au même Trump, en dépit de ses multiples écarts (les évangéliques ont par ailleurs connu leur propre revirement sur l'importance des turpitudes personnelles des leaders, condamnées fermement pour Clinton, mises au second plan s'agissant de Trump). 
Pour Mason, cette évolution (alignement des marqueurs identitaires et sociaux par parti) sur les six dernières décennies aux États-Unis résulte de trois phénomènes majeurs :

- le premier est le grand réalignement partisan des années 1960, qui a fait du Sud la base républicaine et a conduit à la quasi-disparition des démocrates du Sud (pour mémoire, Nixon comprend le potentiel en 1968, quatre ans après le passage de la loi sur les droits civiques au Congrès, loi poussée par un président démocrate, Lyndon Johnson, et votée par un Congrès démocrate, avec sa Southern Strategy, visant à reprendre le Sud aux démocrates - qui y étaient dominants depuis la guerre de Sécession ${ }^{4}$ );

- le second phénomène est l'alliance de la droite religieuse et du parti républicain dans les années 1980, et qui a conduit en 2000 à l'inclusion du «Contrat avec la famille américaine » (sorte de plateforme politique de la coalition chrétienne) dans le programme républicain en 2000: autre grand signal, dont certains passages du livre de J.D. Vance donnent une illustration frappante, et qui se traduit par le soutien des chrétiens évangéliques blancs à Trump à plus de $80 \%$. Or ce mouvement s'est fait parallèlement à la troisième évolution majeure de la société américaine, le déclin de l'engagement des Américains dans toutes les formes d'organisations civiques, associatives, communautaires... résultat d'une combinaison de facteurs (mondialisation, désindustrialisation, politique de prêts immobiliers, évolutions urbaines et para-urbaines). Le déclin frappant d'un engagement qui avait fait l'admiration de Tocqueville a produit une désagrégation du tissu et du lien social, magistralement décrite dans le livre du sociologue Robert Putnam paru en 2000, Bowling Alone. Mason considère que c'est cette désagrégation qui a facilité la réorganisation (le tri) et l'alignement des diverses identités sociales, culturelles et religieuses déjà évoquées (p. 41 et suivantes).

- Cette évolution a été accélérée par deux autres tendances liées entre elles, qui constituent le troisième phénomène, qui est double: un parti démocrate se désintéressant d'un projet collectif global et des droits sociaux des travailleurs pour se concentrer sur des droits dits sociétaux des minorités; le développement dans les années 1980 et 1990 d'un écosystème médiatique conservateur de plus en plus radical, agressif et intolérant. L'apparition des réseaux sociaux à la fin des années 2000 a accentué encore ce phénomène, en raison du principe sur lequel leurs algorithmes ont été bâtis.

9 On a bien là un phénomène de "cercle vicieux " (ou pour reprendre l'expression plus neutre en anglais de "feedback loop on top of feedback loops»), créant une tempête parfaite, une «perfect storm » au niveau de l'opinion américaine, que Trump a su saisir mieux que tous ses rivaux et exploiter pour gagner la nomination républicaine, puis la présidence.

10 Évoquant un article de 2016 du Washington Post, dans lequel l'auteur s'étonne du succès de Trump, dont les positions idéologiques semblent loin du conservatisme américain, Mason explique ainsi (80) que Trump n'a pas déchiré le parti républicain; il a au contraire su s'adresser directement aux différents groupes qui se sont alignés avec le parti républicain dans les années récentes, et il a réussi cela sans se préoccuper du contenu politique, en parlant à leurs identités.

\section{Le tribalisme, stade ultime de la polarisation?}

11 Le livre de Mason est essentiel pour comprendre le tribalisme comme stade ultime de la polarisation politique, qui ne dépend quasiment plus des positions politiques précises. 
12 En effet, le concept de polarisation politique semble a priori renvoyer à une accentuation de l'opposition entre des pôles idéologiques. Or ce que l'élection de Trump a montré, c'est que justement l'idéologie au sens des positions politiques importe peu aux électeurs américains : on l'a vu avec les évolutions spectaculaires sur toutes sortes de sujets, du déficit budgétaire (censé être le principal cheval de bataille du mouvement Tea Party) à l'assurance-santé, en passant par les droits de douane, la Russie, ou encore les frappes en Syrie...

13 Ce que Mason illustre, c'est bien la perte de sens de la "folk theory » de la démocratie, l'idée que les gens votent en fonction de leurs intérêts et valeurs, face à une approche de la politique qui ressemble à celle des supporters sportifs : on est pour son équipe, on veut qu'elle gagne, peu importe comment et sur quelles positions.

14 Comme l'explique Mason (22), tout son propos s'appuie sur la distinction nécessaire entre l'idéologie définie par l'identité ou la combinaison d'identités que certains considèrent comme déterminant leur vision du monde (conception identitaire de l'idéologie donc, si l'on veut) et l'idéologie définie par un ensemble de positions et principes politiques (conception politique de l'idéologie). Or les identités sont globalisantes, déterministes, plus faciles à attaquer, instrumentaliser et mobiliser. Les conséquences politiques sont immenses.

15 À noter que cet alignement des identités s'est fait d'abord au sein du parti républicain, sous l'impulsion (tactique politique) de ses dirigeants, un constat largement partagé ${ }^{\text {. }}$ Rappelons ici le phénomène des "Reagan Democrats ", ces voix démocrates qui (déjà) dans les années 1980 s'étaient portées sur Reagan plutôt que sur Carter ou surtout Mondale, et qui venaient déjà pour beaucoup de la Rust Belt, foyer de cette classe populaire blanche qui a massivement voté Trump en 2016.

16 J.D. Vance et son Élégie des Hillbilly l'illustre à merveille (Hillbilly peut se traduire par "plouc» ou "péquenaud» de la région des Appalaches; un terme synonyme couramment utilisé mais plus large géographiquement est « White Trash », littéralement " déchet blanc », ce qui en dit long). Le livre décrit l'enfance et la jeunesse de l'auteur dans la grande région des Appalaches, entre le Kentucky et l'Ohio, et évoque 1984, la seule fois de sa vie où son grand-père (papaw) a voté républicain (le grand-père est mort avant Trump) :

I was born in late summer 1984, just a few months before Papaw cast his first and only vote for a Republican: Ronald Reagan. Winning large blocks of Rust Belt Democrats like Papaw, Reagan went on to the biggest electoral landslide in modern American history. "I never liked Reagan much," Papaw later told me. "But I hated that son of a bitch Mondale." Reagan's Democratic opponent, a well-educated Northern liberal, stood in stark cultural contrast to my hillbilly Papaw. Mondale never had a chance, and after he departed from the political scene, Papaw never again voted against his beloved "party of the workingman."

Or l'identité (raciale, religieuse, culturelle), a fortiori les identités d'un individu, si elles sont alignées et confondues avec l'appartenance partisane, transforment les règles du jeu politique sur plusieurs points. En effet, elles sont plus facilement mobilisables, attaquables, et instrumentalisables, le tout indépendamment des opinions politiques de départ. C'est ce que Mason démontre en utilisant les apports de la psychologie sociale (61) : quand les identités sont alignées dans un groupe, les gens de ce groupe sont moins tolérants, plus biaisés et plus en colère contre les gens hors-groupe ${ }^{6}$. 

cet égard :

If everything -the Supreme Court, the fate of Western civilization, the survival of the planet- depends on tribal victory, then neither individuals nor ideas can be determinative. Mason montre également la manière dont la droite religieuse, jusque-là non affiliée politiquement, a franchi le pas en 1989 après l'échec de la candidature du télévangéliste extrémiste Pat Robertson, en créant la Coalition chrétienne (33 et suivantes). Associée à la campagne républicaine historique de Newt Gingrich en novembre 1994, la Coalition chrétienne participe donc à la reconquête républicaine du Congrès, après quarante ans dans la minorité (1954). C'est un moment de basculement de la vie politique américaine contemporaine qui mène au climat actuel et à Trump: dès 2000, le programme politique de la droite évangélique est intégré à la plate-forme du parti républicain.

Citons là encore Hillbilly Elegy, quand l'auteur se rapproche de son père qui est un chrétien évangélique fervent: ce passage éclaire ce qu'a signifié concrètement le ralliement de la droite religieuse militante au parti républicain, en termes de ressenti et de vote (96):

The deeper I immersed myself in evangelical theology, the more I felt compelled to mistrust many sectors of society. Evolution and the Big Bang became ideologies to confront, not theories to understand. [...] In my new Church, I heard more about the gay lobby and the war on Christmas than about any particular character trait that a Christian should aspire to have. [...] Morality was defined by not participating in this or that particular social malady: the gay agenda, evolutionary theory, Clintonian liberalism, or extramarital sex.

The world lurched toward moral corruption -slouching toward Gomorrah. The Rapture was coming, we thought. Apocalyptic imagery filled the weekly sermons and the Left Behind books (one of the best-selling fiction series of all time). [...] The End Times were the natural finish for a culture sliding so quickly toward the abyss.

21 On relèvera l'importance de la pensée apocalyptique dans cette influence évangélique, que d'autres ont décrite comme l'apport américain spécifique dans la transplantation du protestantisme outre-Atlantique, et que l'on retrouve aujourd'hui aussi bien chez les candidats républicains que sur Fox News?

Terminons sur cette évocation de Barack Obama par J.D. Vance, sur la signification, audelà du racisme, de l'accession au pouvoir du premier président noir pour ces anciens électeurs démocrates de la Rust Belt (191-192) :

President Obama came on the scene right as so many people in my community began to believe that the modern American meritocracy was not built for them. [...] Obama strikes at the heart of our deepest insecurities. He is a good father while many of us aren't. He wears suits to his job while we wear overalls, if we're lucky enough to have a job at all. His wife tells us that we should be feeding our children certain foods, and we hate her for it - not because we think she's wrong, but because we know she's right.

Pour conclure, ces deux livres non seulement expliquent et illustrent le tribalisme, ils éclairent également le débat récurrent sur les déterminants du vote populiste (pour simplifier : l'économie ou le racisme), déjà plus ou moins tranché avec la notion de " sentiment de perte de statut » qui englobe les déterminants économiques et culturels ${ }^{8}$ - en somme, ce qui définit les classes sociales. En ce sens, la victoire de Trump signe bien le recul du rêve américain et le retour de la lutte des classes aux États-Unis. 
24 Mais cette lutte des classes est différente en raison de sa dimension identitaire, mise en évidence par la notion de tribalisme. C'est bien une crise existentielle que traversent les États-Unis, que seul le politique peut résoudre -l'alternative étant non pas une " guerre de sécession » mais plutôt une dissociation progressive qui serait marquée par des points de friction récurrents pouvant dégénérer en affrontements armés, de toute façon déjà fréquents. Or pour l'instant la vie politique américaine est dysfonctionnelle, les institutions réagissant mal à la recomposition partisane contemporaine: la polarisation débouche le plus souvent sur la paralysie ou la contradiction (c'est frappant en politique étrangère). Crise institutionnelle ? Pas encore, mais le face-à-face Trump-Pelosi est fascinant à observer et sera riche de conséquences. Quoi qu'il en soit, la division du pays est profonde, et l'état de crise politique permanente devrait durer.

\section{NOTES}

1. Une recherche Google « tribalism in the US » donne 1590000 résultats.

2. Voir ce titre d'une conférence de Paul Ryan à l'AEI en septembre 2018: "Constitutional government in an age of tribalism and identity politics. "

3. Voir par exemple le tout juste paru Hidden Tribes: A Study of America's Polarized Landscape, Stephen Hawkins, Daniel Yudkin, Miriam Juan-Torres, Tim Dixon, New York, More in Common, 2018.

4. Pour un résumé voir Maya Kandel, Les États-Unis et le monde, de George Washington à Donald Trump, Paris, Perrin, 2018, chapitre 6 (« Le grand réalignement », p. 126-129).

5. Voir aussi le livre de Thomas Mann et Norman Ornstein, It's Even Worse Than It Looks, New York, Basic Books, 2016.

6. Ainsi, quelqu'un qui est irlandais et catholique sera a priori plus intolérant que quelqu'un qui est irlandais et juif.

7. Voir le livre essentiel de Matthew Avery Sutton, American Apocalypse: A History of Modern Evangelicalism, Harvard University Press, 2014.

8. Ronald Ingelhart et Pippa Norris, "Trump, Brexit and the Rise of Populism: Economic HaveNots and Cultural Backlash", Harvard Kennedy School Faculty Research Working Papers, 2017. Voir aussi cet article de Maggie Habermann dans le New York Times du 14 octobre 2018: "He's 'One of Us': The Undying Bond Between the Bible Belt an Trump": "I don't really look at him as a politician", he said. "Even now, I look at him as just one of us. He doesn't act like he's above you, as a person." 


\section{AUTEURS}

\section{MAYA KANDEL}

Docteure en histoire, diplômée de Columbia University (SIPA) et de Sciences Po Paris, Maya Kandel est chercheuse associée à l'Université Sorbonne Nouvelle - Paris 3 (laboratoire CREW). Elle travaille actuellement au Centre d'Analyse, de Prévision et de Stratégie (CAPS) du Ministère de l'Europe et des Affaires Etrangères. De 2011 à 2016, elle a dirigé le programme sur les ÉtatsUnis à l'IRSEM. Son dernier livre, Les États-Unis et le monde, de George Washington à Donald Trump, est paru en 2018 aux Éditions Perrin. 